\title{
Bit storage and bit flip operations in an electromechanical oscillator
}

I. MAHBOOB AND H. YAMAGUCHI

Nature Nanotechnology 3, 275-279 (2008)

There were two errors in thirteenth paragraph of this letter. The relation from which the power consumption can be extracted should read $C V^{2} f_{s}$, and the actual expression for the power consumed should read $m \omega^{3} x^{2} / 2 \pi Q$. These errors have also been corrected in the supplementary information.

\section{Improved nanofabrication through guided transient liquefaction}

STEPHEN Y. CHOU AND QIANGFEI XIA

Nature Nanotechnology 3, 295-300 (2008)

There was an error in the fourth paragraph of the section "Applying SPEL to metals and semiconductors". The first sentence should read: "In guided-SPEL, local spacers are used to keep a plate fixed above the nanostructures (Fig. 1), and the molten nanostructures rise up against surface tension until they reach the plate." 